\title{
Nucleation phenomena in electrochemical systems: kinetic models
}

\author{
Alexander Milchev ${ }^{1,2}$ (i)
}

Received: 21 May 2015/Accepted: 22 December 2015/Published online: 8 February 2016

(C) Springer International Publishing Switzerland 2016

\begin{abstract}
The classical thermodynamics allow deriving theoretical expressions for the nucleation work and for the size of the critical nuclei in a supersaturated system. However, information on the rate of nucleus formation should be determined by means of kinetic considerations. This article sheds light on the nucleation kinetics in electrochemical systems at a constant thermodynamic supersaturation $\Delta \tilde{\mu}$. Theoretical expressions are derived for the stationary and non-stationary nucleation rate.
\end{abstract}

Keywords Nucleation rate Active sites - Induction time

\section{List of symbols: kinetic models}

$\begin{array}{ll}\alpha & \text { Ion's transfer coefficient } \\ a_{\infty}^{\text {sol }} & \text { Ionic activity of the electrolyte solution } \\ e & \text { Elementary electric charge } \\ E & \text { Electrode potential } \\ I_{\mathrm{st}} & \text { Stationary nucleation rate } \\ k & \text { Boltzmann constant } \\ T & \text { Absolute temperature } \\ n & \text { Number of atoms in a cluster of the new phase } \\ N_{0} & \text { Number of sites on the electrode surface } \\ U_{\mathrm{i}}, U_{\mathrm{i}} * & \text { where nucleus formation takes place } \\ \Gamma & \text { "Chemical" parts of energy barriers }\end{array}$

Alexander Milchev

amilchev@gmail.com; amilchev@ipc.bas.bg

1 Evgeni Budevski Institute of Electrochemistry and Energy Systems, Bulgarian Academy of Sciences, Acad. G. Bonchev Str. B1. 10, 1113 Sofia, Bulgaria (Regular Member)

2 Rostislav Kaischev Institute of Physical Chemistry, Bulgarian Academy of Sciences, 1113 Sofia, Bulgaria (Associate Member) $\varphi_{1 / 2} \quad$ Separation work of a single atom from the half crystal position

$\varphi_{n}{ }^{*} \quad$ Separation work of the nth atom from an $n$ atomic cluster

$\varphi_{i}{ }^{*} \quad$ Separation work of a single atom from the ith site of the electrode surface

$K_{a}^{+}, K_{a}^{-} \quad$ Frequencies of appearance and disappearance of free active sites to and from the electrode surface

$\omega_{+n}, \omega_{-n} \quad$ Frequencies of attachment and detachment of a single atom to and from an $n$-atomic cluster

$Z_{a}(t) \quad$ Number of free active sites on the electrode surface

$Z_{n}(t) \quad$ Number of $n$-atomic clusters

\section{Introduction}

Theoretical considerations of the nucleation kinetics are usually performed assuming that the process of nucleus formation is a set of consecutive bimolecular reactions of the type $a_{n-1}+a_{1} \rightarrow a_{n}$ where $a_{1}$ is the monomer and $a_{n-1}$ and $a_{n}$ are clusters of the new phase consisting of $n-1$ and of $n$ atoms, respectively. Thus, bearing in mind that an $n$ atomic cluster of the new phase can be formed either by attaching a single atom to the $(n-1)$-atomic cluster or by detaching a single atom from the $(n+1)$-atomic one, the time dependence of the number $Z_{n}(t)$ of the $n$-atomic clusters on the electrode surface is expressed as [1] (see also the references cited therein).

$$
\begin{aligned}
\frac{\mathrm{d} Z_{n}(t)}{\mathrm{d} t}= & {\left[\omega_{+n-1} Z_{n-1}(t)-\omega_{-n} Z_{n}(t)\right] } \\
& -\left[\omega_{+n} Z_{n}(t)-\omega_{-n+1} Z_{n+1}(t)\right]
\end{aligned}
$$

$n=1,2,3 \ldots$ 
Here $\omega_{+n-1} / \mathrm{s}^{-1}$ and $\omega_{+n} / \mathrm{s}^{-1}$ are the frequencies of attachment of single atoms to clusters consisting of $n-1$ and of $n$ atoms, respectively, $\omega_{-n}$ and $\omega_{-n+1}$ are the frequencies of detachment of single atoms from clusters consisting of $n$ and of $n+1$ atoms and $Z_{n-1}(t) / \mathrm{cm}^{-2}, Z_{n}(t) /$ $\mathrm{cm}^{-2}$ and $Z_{n+1}(t) / \mathrm{cm}^{-2}$ are the number densities of $(n-1)-$, $n$ - and $(n+1)$-atomic clusters on the electrode surface at time $t$.

\section{Stationary nucleation kinetics: a general approach}

In case of a constant supersaturation $\Delta \tilde{\mu}$ a stationary state is established in the electrochemical system after a certain induction period. Thus, the number $Z_{n}$ of the $n$-atomic clusters is no more a time-dependent quantity, i.e., $\mathrm{d} Z_{n}(t) / \mathrm{d} t=0$ and Eq. (1) turns into

$\omega_{+n-1} Z_{n-1}-\omega_{-n} Z_{n}=\omega_{+n} Z_{n}-\omega_{-n+1} Z_{n+1}=I_{\mathrm{st}}$

which defines a constant, stationary nucleation rate $I_{\mathrm{st}}$.

Rewriting Eq. (2) for all values of $n$ from $n=1$ to $n=s-1$ one obtains the following set of simple algebraic equations:

$I_{\mathrm{st}}=\omega_{+0} N_{0}-\omega_{-1} Z_{1}$
$I_{\mathrm{st}}=\omega_{+1} Z_{1}-\omega_{-2} Z_{2}$
$\vdots$
$I_{\mathrm{st}}=\omega_{+n-1} Z_{n-1}-\omega_{-n} Z_{n}$
$\quad \cdot \cdot \cdot$
$I_{\mathrm{st}}=\omega_{+s-1} Z_{s-1}-\omega_{-s} Z_{s}$

$N_{0}$ being the number of sites on the electrode surface where the nucleus formation takes place with a measurable rate. It was solved firstly by Becker and Döring [2] already in 1935 under the simplifying assumption that the $s$-atomic clusters of the new phase were disintegrated to single atoms and returned back to the parent phase (in our case the electrolyte solution) to preserve its initial state and, therefore, $Z_{s}=0$. Thus, the following expression was obtained for the stationary nucleation rate

$I_{\mathrm{st}}=\frac{N_{0}}{\frac{1}{\omega_{+0}}+\frac{\omega_{-1}}{\omega_{+0} \omega_{+1}}+\frac{\omega_{-1} \omega_{-2}}{\omega_{+0} \omega_{+1} \omega_{+2}}+\cdots+\frac{\omega_{-1} \omega_{-2} \omega_{-3} \ldots \omega_{-n}}{\omega_{+0} \omega_{+1} \omega_{+2} \omega_{+3} \ldots \omega_{+n}}+\ldots}$

or

$I_{\mathrm{st}}=\frac{N_{0}}{\sum_{0}^{s-1} \frac{1}{\omega_{+n}} \frac{\omega_{-1} \omega_{-2} \omega_{-3} \ldots \omega_{-n}}{\omega_{+0} \omega_{+1} \omega_{+2} \ldots \omega_{+n-1}}}$

Note that in this general form Eq. (4) applies without restrictions to all cases of nucleus formation in various physical systems, of course, if the quantities $\omega_{+}$and $\omega_{-}$are defined in a proper way. An explicit theoretical formula for $I_{\text {st }}$ in electrochemical systems revealing the supersaturation dependence of this most important quantity is derived in the next section.

\section{Stationary nucleation kinetics}

In case of electrochemical phase formation, the frequencies $\omega_{+\mathrm{i}}$ and $\omega_{-\mathrm{i}}$ of single ions' direct attachment and detachment ${ }^{1}$ to and from the $i$ th site of the electrode surface at an electrode potential $E$ are given by the following theoretical expressions [1, 3-8]:

$\omega_{+i}=k_{+i} a_{\infty}^{\mathrm{sol}} \exp \left(-U_{i} / k T\right) \exp (-\alpha z e E / k T)$

$\omega_{-i}=k_{-i} \exp \left(-U_{i}^{*} / k T\right) \exp [(1-\alpha) z e E / k T]$

Here $k_{+i}$ and $k_{-i}$ are frequency factors, $a_{\infty}^{\text {sol }}$ is the ionic activity of the electrolyte solution, $\alpha$ is the ions transfer coefficient, $U_{i}$ and $U_{i}^{*}$ are the "chemical" parts of the energy barriers to transfer an atom to and from the $i$ th site of the electrode surface and the difference $U_{i} *-U_{i}$ defines the atom's separation work $\varphi_{i} *$ from the $i$ th site. The other symbols have the same meanings as in Ref. [9].

Suppose now that the $i$ th site of the electrode surface is a cluster of the new phase consisting of $n-1$ atoms. In this case the attachment of a single atom to the $i$ th site is an attachment of a single atom to the $n-1$-atomic cluster and, therefore, the frequency $\omega_{+i}$ coincides with the frequency $\omega_{+n-1}$ from Eqs. (2, 3 and 4). At the same time, the detachment of a single atom from the $i$ th site is, in fact, detachment of one atom from its $n-1$ neighboring atoms and, therefore, the frequency $\omega_{-i}$ corresponds to the frequency $\omega_{-n}$ in Eqs. (2, 3 and 4). Thus, the ratio $\omega_{-i} / \omega_{+i}$ coincides with the ratio $\omega_{-n} / \omega_{+n-1}$ from the theory of Becker and Döring [2] and is given by

$\frac{\omega_{-n}}{\omega_{+n-1}}=\frac{\xi_{n}}{a_{\infty}^{\text {sol }}} \exp \left(-\frac{\varphi_{n}^{*}}{k T}\right) \exp \left(\frac{z e E}{k T}\right)$

where $\xi_{n}=k_{-n} / k_{+n-1}$.

Consider now a bulk metal crystal dipped in the electrolyte solution of metal ions with activity $a_{\infty}^{\text {sol }}$ and kept at a potential $E$. In this case the frequencies of attachment and detachment $\omega_{+1 / 2}$ and $\omega_{-1 / 2}$ of a single atom to and from the half crystal position are expressed as [1],

$\omega_{+1 / 2}=k_{+1 / 2} a_{\infty}^{\mathrm{sol}} \exp \left(-U_{1 / 2} / k T\right) \exp |-(\alpha z e E / k T)|$

$\omega_{-1 / 2}=k_{-1 / 2} \exp \left(-U_{1 / 2}^{*} / k T\right) \exp [(1-\alpha) z e E / k T]$

where the difference $U_{1 / 2} *-U_{1 / 2}=\varphi_{1 / 2}$ gives the separation work of an atom from the half crystal position [9].

\footnotetext{
${ }^{1}$ Surface diffusion of atoms adsorbed on the working electrode is not considered in this article. Information on this subject can be found in Ref. [1].
} 
Apparently, a state of stable thermodynamic equilibrium is established in this electrochemical system at a potential $E=E_{\infty}$ at which the condition $\omega_{+1 / 2}\left(E_{\infty}\right)=\omega_{-1 / 2}\left(E_{\infty}\right)$ is fulfilled and from Eqs. $(8,9)$ one obtains,

$\frac{\omega_{-1 / 2}\left(E_{\infty}\right)}{\omega_{+1 / 2}\left(E_{\infty}\right)}=\frac{\xi_{1 / 2}}{a_{\infty}^{\text {sol }}} \exp \left(-\varphi_{1 / 2} / k T\right) \exp \left(z e E_{\infty} / k T\right)=1$

where $\xi_{1 / 2}=k_{-1 / 2} / k_{+1 / 2}$. Taking the logarithm of this expression it transforms into the equation of Nernst for the equilibrium potential $E_{\infty}$, now derived by means of kinetic considerations:

$E_{\infty}=E_{0, s}+\frac{k T}{z e} \ln a_{\infty}^{\mathrm{sol}}$

Here $E_{0, \mathrm{~s}}=\frac{1}{z e}\left(\varphi_{1 / 2}-k T \ln \xi_{1 / 2}\right)$ is the standard state electrode potential at an activity $a_{\infty}^{\text {sol }}=1$.

Bearing in mind that according to Eq. (10) the activity $a_{\infty}^{\text {sol }}$ is given by

$a_{\infty}^{\text {sol }}=\xi_{1 / 2} \exp \left(-\varphi_{1 / 2} / k T\right) \exp \left(z e E_{\infty} / k T\right)$

and substituting the last expression in Eq. (7), introducing also the supersaturation $\Delta \tilde{\mu}=z e\left(E_{\infty}-E\right)=z e \eta$, for the frequency ratio $\omega_{-n} / \omega_{+n-1}$ one obtains

$$
\begin{aligned}
\frac{\omega_{-n}}{\omega_{+n-1}} & =\frac{\xi_{n}}{\xi_{1 / 2}} \exp \left(\frac{\varphi_{1 / 2}-\varphi_{n}^{*}}{k T}\right) \exp \left(-\frac{z e \eta}{k T}\right) \\
& =\exp \left(\frac{\tilde{\mu}_{n}^{*}-\tilde{\mu}_{1 / 2}}{k T}\right) \exp \left(-\frac{\Delta \tilde{\mu}}{k T}\right)
\end{aligned}
$$

Thus, for the product $\frac{\omega_{-1} \omega_{-2} \omega_{-3} \ldots \omega_{-n}}{\omega_{+0} \omega_{+1} \omega_{+2} \ldots \omega_{+n}}$ it results:

$$
\begin{aligned}
\frac{\omega_{-1} \omega_{-2} \omega_{-3} \ldots \omega_{-n}}{\omega_{+0} \omega_{+1} \omega_{+2} \ldots \omega_{+n}} & =\frac{1}{\omega_{+n}} \exp \left[\frac{-n \Delta \tilde{\mu}+\sum_{1}^{n} \tilde{\mu}_{i}^{*}-n \tilde{\mu}_{1 / 2}}{k T}\right] \\
& =\frac{1}{\omega_{+n}} \exp \left[\frac{\Delta \widetilde{G}_{(n)}}{k T}\right]
\end{aligned}
$$

where $\sum_{1}^{n} \tilde{\mu}_{i}^{*}-n \tilde{\mu}_{1 / 2}=\tilde{\Phi}(n)$ accounts for the difference between the Gibbs free energies of $n$ atoms when they form an $n$-atomic nucleus on the working electrode $\left(\sum_{1}^{n} \tilde{\mu}_{i}^{*}\right)$ and when they belong to a bulk crystal of the new phase $\left(n \tilde{\mu}_{1 / 2}\right)$ [see also Ref. [9], Eq. (20)].

The above theoretical formula Eq. (14) tells us that each term in the denominator of Eq. (4) is an exponential function of the nucleation work $\Delta \tilde{G}(n)$ of a given $n$-atomic cluster of the new phase. Apparently this is valid also for the term $1 / \omega_{+0}$, which can be rewritten as $\left(1 / \omega_{+0}\right) \exp [\Delta \tilde{G}(0) / k T]=1 / \omega_{+0} \quad[1] \quad$ since the work $\Delta \tilde{G}(0)$ to form a zero-atomic cluster on the electrode surface equals zero, too, and $\exp [\Delta \tilde{G}(0) / k T]=1$. Therefore, the general theoretical expression for the stationary nucleation rate $I_{\mathrm{st}}$ can be rewritten as [1]:
$I_{\mathrm{st}}=\frac{N_{0}}{\sum_{0}^{s-1} \frac{1}{\omega_{+n}} \exp \left[\frac{\Delta \tilde{G}(n)}{k T}\right]}$

The next step in the theoretical considerations of the nucleation kinetics consists in the derivation of an explicit theoretical formula for the stationary nucleation rate which reveals its supersaturation dependence and the section that follows describes the classical approach to this problem.

\section{Classical nucleation theory}

The classical theory of nucleus formation operates with macroscopic physical quantities applicable to sufficiently large clusters of the new phase so that their size $n$ can be considered as a continuous variable and the work for nucleus formation $\Delta \tilde{G}(n)$ as a differentiable function. Thus, the sum in Eq. (15) can be replaced by an integral the solution of which yields $[11,12]$ :

$I_{\mathrm{st}}=N_{0} \omega_{+n_{c}} \Gamma \exp \left[-\frac{\Delta \tilde{G}\left(n_{c}\right)}{k T}\right]$

Here $\Gamma=\left[\Delta \tilde{G}\left(n_{c}\right) / 3 \pi n_{c}^{2} k T\right]^{1 / 2}$ is the so-called nonequilibrium Zeldovich factor, which accounts for the difference between the quasi-equilibrium and the stationary number of critical nuclei at the corresponding supersaturation.

Assuming that three-dimensional nuclei form on the electrode surface and accounting for the overpotential dependence of $\omega_{+n_{c}}, \Gamma$ and $\Delta \tilde{G}\left(n_{c}\right)$ [1,9] the formula for the stationary nucleation rate $I_{\mathrm{st}}$ can be rewritten as

$I_{\mathrm{st}}=K_{1} \exp (\alpha z e \eta / k T) \exp \left[-\frac{K_{2}}{\eta^{2}}\right]$

where the constant $K_{1}$ is determined by the flux of ions' impingements to the critical nucleus surface ${ }^{2}$ and $K_{2}=\Delta \tilde{G}\left(n_{c}\right) \eta^{2} / k T$.

Dividing the two parts of Eq. (17) by $\exp (\alpha z e \eta / k T)$ one obtains

$M_{3 D}=K_{1} \exp \left[-\frac{K_{2}}{\eta^{2}}\right]$

where $M_{3 \mathrm{D}}=I_{\mathrm{st}} \exp (-\alpha z e \eta / k T)$. Thus, taking the logarithm of Eq. $\left(17^{\prime}\right)$ the latter transforms into a linear $\ln M_{3 \mathrm{D}}$ versus $\eta^{-2}$ relationship:

$\ln \mathrm{M}_{3 \mathrm{D}}=\ln K_{1}-\frac{K_{2}}{\eta^{2}}$

the slope of which $\mathrm{d}\left(\ln M_{3 \mathrm{D}}\right) / d \eta^{-2}=-K_{2}$ allows the determination of both the nucleation work $\Delta \tilde{G}\left(n_{c}\right)$ and the

\footnotetext{
${ }^{2}$ Analytical formulas for $K_{1}$ in case of solid and liquid clusters of the new phase can be found in Ref. [1].
} 
size $n_{c}$ of the critical nucleus at any value of $\eta$ according to:

$\Delta \tilde{G}\left(n_{c}\right)=\frac{k T}{\eta^{2}} K_{2}$

$n_{c}=\frac{2 k T}{z e \eta^{3}} K_{2}$

What follows presents some experimental data for the stationary nucleation rate obtained in case of silver electrodeposition on a platinum single crystal electrode [8].

Direct studies of electrochemical nucleation kinetics are based on examination of the "number $N(t)$ of nuclei vs. time" relationship by means of a pulse potentiostatic technique, which consists in the following (Fig. 1).

A triple potentiostatic pulse is applied to the electrodes of an electrochemical cell containing a solution of metal ions, the cathodic overpotential $\eta_{n}$ of the first one being sufficiently high to initiate a process of nucleus formation on the working electrode. During the second pulse the nuclei grow at a lower cathodic overpotential $\eta_{g}$ to microscopically visible sizes (Fig. 2) and can be easily counted. Finally, a third, anodic pulse $\eta_{d}$ is applied to dissolve the nuclei formed, thus preparing the electrode surface for the next triple pulse series.

Most generally, the quantity $N(t)$ is defined as $N(t)=$ $\int_{0}^{t} J(t) d t$ since in real physical systems the rate of nucleus formation $J(t)=\mathrm{d} N(t) / \mathrm{d} t$ could be a time-dependent quantity. In case of a stationary nucleation process, i.e., constant nucleation rate $I_{\mathrm{st}}, N(t)=I_{\mathrm{st}} t$ and, therefore, one should expect linear $N(t)$ vs $t$ relationships. However, Fig. 3 clearly shows that a stationary state is established after a certain induction period and in such cases the stationary nucleation rate $I_{\mathrm{st}}$ should be determined from the slopes of the linear portions of the experimental $N(t)$ curves obtained at different overpotentials $\eta$.

Figure 4 shows a linear $\ln M_{3 D}$ vs $\eta^{-2}$ relationship demonstrating an excellent qualitative agreement between

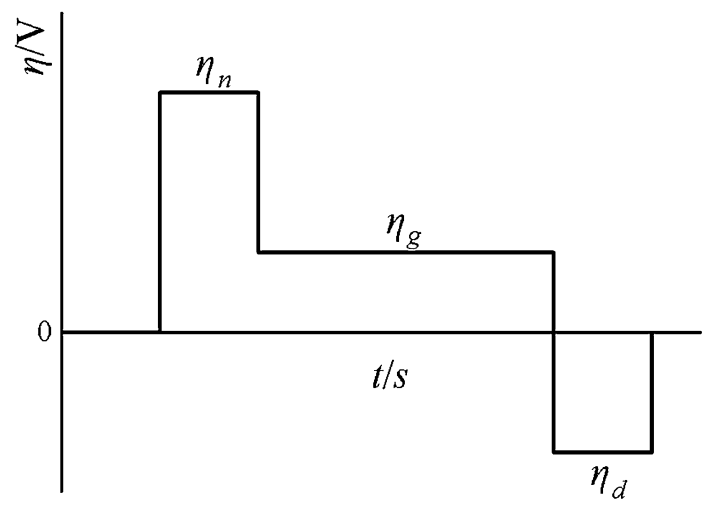

Fig. 1 Triple pulse potentiostatic technique (a schematic representation) the theoretical Eq. (18) and experimental data. However, interpretation of the constant $K_{2}$ based on Eqs. (19 and 20) leads to unexpected results-it turns out that the size $n_{c}$ of the critical silver nuclei varies from 6 to 3 atoms within the whole overpotential interval $0.175-0.205 \mathrm{~V}$. Apparently this excludes a reliable theoretical analysis of these experimental data based on the classical nucleation theory and the reason is that the latter operates with macroscopic physical quantities like surface and volume, which have no physical significance for clusters consisting of several atoms.

It must be noted that such a crucial contradiction between the macroscopic classical nucleation theory and experimental data was observed in various physical systems and this is what led to the development of the atomistic nucleation theory, firstly, in case of nucleus formation from a vapor phase [15-17] and then also in case of electrochemical nucleation on a foreign substrate [4-8] (see also Ref. [1] and the references cited therein).

\section{Atomistic nucleation theory}

In case of small clusters $(n<10)$, the size $n$ cannot be considered as a continuous variable, the work for nucleus formation $\Delta \tilde{G}(n)$ is not a differentiable function and the sum in Eq. (15) cannot be replaced by an integral. However, the term corresponding to the critical nucleus consisting of $n_{c}$ atoms is much bigger than all the rest and the general formula for the stationary nucleation rate (Eqs. 4, $\left.4^{\prime}\right)$ can be rewritten as [1]:

$I_{\mathrm{st}}=\frac{N_{0}}{\chi \frac{\omega_{-1} \omega_{-2} \omega_{-3} \ldots \omega_{-n_{c}}}{\omega_{+0} \omega_{+1} \omega_{+2} \omega_{+3} \ldots \omega_{+n_{c}}}}=N_{0} \frac{1}{\chi} \omega_{+n_{c}} \exp \left[-\frac{\Delta \tilde{G}\left(n_{c}\right)}{k T}\right]$

Here the quantity $\chi$ accounts for the contribution of all terms corresponding to clusters different from the critical $n_{c}$-atomic one. Thus, using the general formula for the nucleation work $\Delta \tilde{G}\left(n_{c}\right)=-n_{c} \Delta \tilde{\mu}+\tilde{\Phi}\left(n_{c}\right)$ and expressing the frequency $\omega_{+n_{c}}$ of a single ion attachment to the critical nucleus as $\omega_{+n_{c}}=k_{+n_{c}} a_{\infty}^{\text {sol }} \exp \left(-U_{n_{c}} / k T\right) \exp \left[-\alpha z e\left(E_{\infty}-\right.\right.$ $\eta) / k T]$, Eq. (21) transforms into [1]:

$I_{\mathrm{st}}=K^{*} \exp \left[\frac{\left(n_{c}+\alpha\right) z e \eta}{k T}\right]$

where

$K^{*}=N_{0} \frac{1}{\chi} k_{+n_{c}} a_{\infty}^{\text {sol }} \exp \left(-\frac{U_{n_{c}}+\alpha z e E_{\infty}}{k T}\right) \exp \left[-\frac{\tilde{\Phi}\left(n_{c}\right)}{k T}\right]$

Figure 5 shows the data for the stationary nucleation rate $I_{\mathrm{st}}$ determined from the linear portions of the $N(t)$ relationships (Fig. 3), now plotted in semi-logarithmic 
Fig. 2 a $\mathrm{Ag}_{7} \mathrm{NO}_{11}$ crystals [13] and b copper crystals [14] electrochemically deposited on a mechanically polished glassy carbon electrode. Magnification $500 \mathrm{X}$
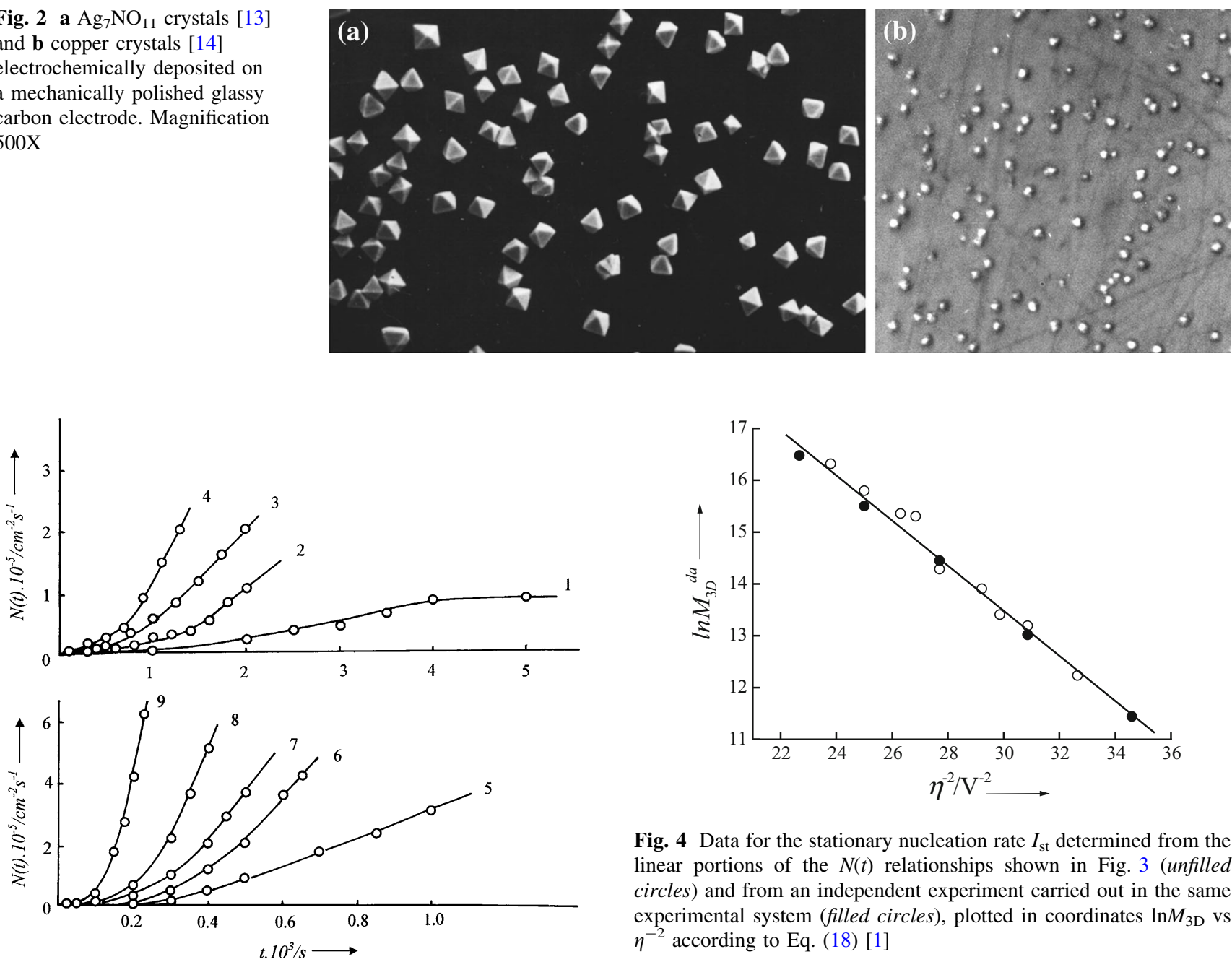

Fig. 4 Data for the stationary nucleation rate $I_{\mathrm{st}}$ determined from the linear portions of the $N(t)$ relationships shown in Fig. 3 (unfilled circles) and from an independent experiment carried out in the same experimental system (filled circles), plotted in coordinates $\ln M_{3 \mathrm{D}}$ vs $\eta^{-2}$ according to Eq. (18) [1]

Fig. 3 Experimental $N(t)$ vs $t$ relationships for silver electrodeposition on a platinum single crystal electrode at various electrochemical overpotentials (1) $\eta=0.175 \mathrm{~V}$, (2) $\eta=0.180 \mathrm{~V}$, (3) $\eta=0.183 \mathrm{~V}$, (4) $\eta=0.185 \mathrm{~V}$, (5) $\eta=0.190 \mathrm{~V}, \quad$ (6) $\eta=0.193 \mathrm{~V}, \quad$ (7) $\eta=0.195 \mathrm{~V}$, (8) $\eta=0.200 \mathrm{~V}$, (9) $\eta=0.205 \mathrm{~V}$ [8]

coordinates $\ln I_{\text {st }}$ vs $\eta$ according to Eq. (22). As seen, a broken straight line is obtained, the slopes $\mathrm{d} \ln I_{\mathrm{st}} / \mathrm{d} \eta$ depending on the size $n_{c}$ of the silver clusters playing the role of critical nuclei in the corresponding supersaturation intervals. Thus, making use of Eq. (22) for $n_{c}$ one obtains:

$n_{c}=\frac{k T}{z e} \frac{d \ln I_{\mathrm{st}}}{d \eta}-\alpha$

which results in $n_{c}=5$ atoms within the interval $(0.175-0.193) \mathrm{V}$ and $n_{c}=2$ atoms within the interval (0.193-0.205) V. Apparently, these values confirm the applicability of the atomistic nucleation theory to this particular experimental study since it was developed namely for the case of high supersaturations and small clusters of the electrochemically formed new phase.

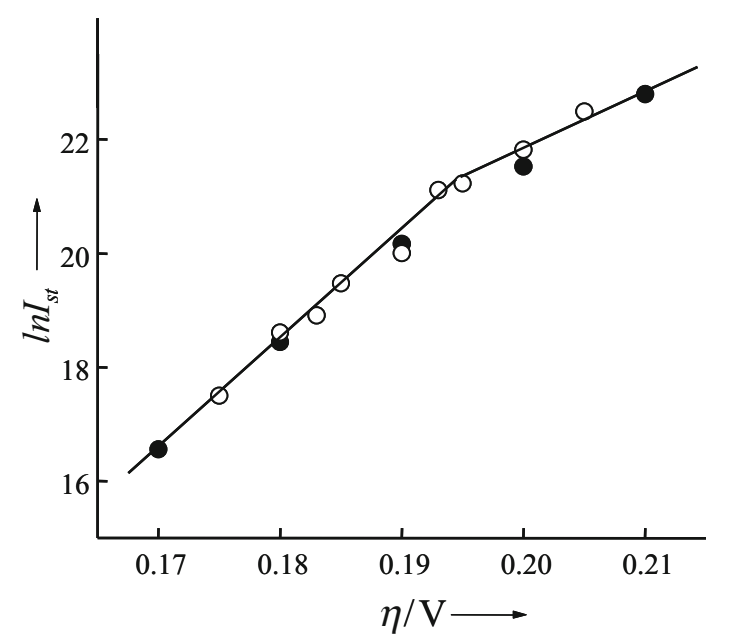

Fig. 5 Data for the stationary nucleation rate of silver on platinum from Fig. 1 plotted in the coordinates of the atomistic theory of electrochemical nucleation

Detailed experimental studies of electrochemical nucleation kinetics performed in wider overpotential intervals can be found in Refs. $[1,8]$. 
The considerations performed thus far clearly show that the supersaturation dependence of the stationary nucleation rate $I_{\mathrm{st}}$ can be schematically presented by the line shown in Fig. 6.

Thus, the fluent curve describes the region of low supersaturations, corresponding to the classical nucleation theory, whereas the broken straight line describes the region of high supersaturations at which clusters consisting of $m_{c}, n_{c}$ and $p_{c}$ atoms play the role of critical nuclei in neighboring supersaturation intervals. It corresponds to the atomistic theory of nucleus formation. Experimental data demonstrating the broken straight line part of the $\ln I_{s t}$ vs $\eta$ relationship within which three different copper clusters play the role of critical nuclei within neighboring supersaturation intervals can be found in Refs. [1, 8] (see also the references cited therein).

\section{Non-stationary nucleation kinetics}

\section{Classical continuity approach}

As I mentioned above a stationary state in a real physical system is established after a certain induction period (see Fig. 3) and, therefore, it is important to derive a general theoretical expression for the nucleation rate $J(t)$ accounting for the time dependence of this important quantity.

The classical approach to this problem suggested by $\mathrm{Y}$. B. Zeldovich [11] (see also Ref. [12] and the references cited therein) consists in considering the clusters' size $n$ as a

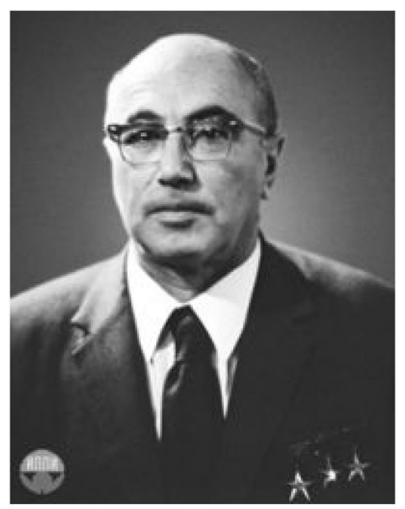

Y. B. Zeldovich

(1914-1987)

continuous variable and expressing the time dependence of the number $Z(n, t)$ of $n$-atomic clusters through the following partial differential equation:

$\frac{\partial Z(n, t)}{d t}=\frac{\partial}{\partial n}\left\{\omega_{+}(n) Z_{e}(n) \frac{\partial}{\partial n}\left[\frac{Z(n, t)}{Z_{e}(n)}\right]\right\}$

Here the product

$J(n, t)=-\omega_{+}(n) Z_{e}(n) \frac{\partial}{\partial n}\left[\frac{Z(n, t)}{Z_{e}(n)}\right]$

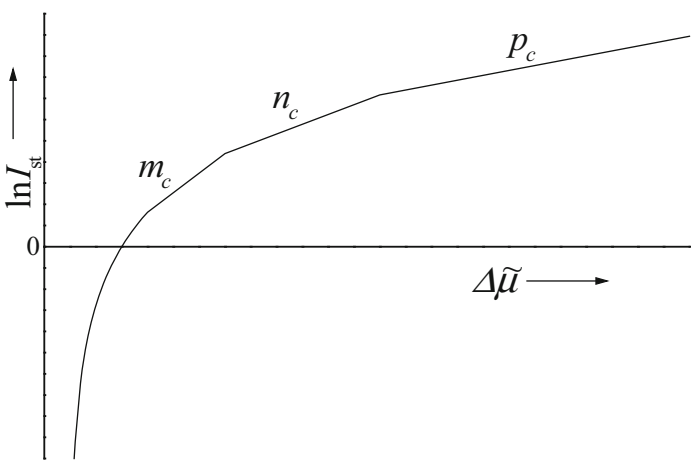

Fig. 6 Supersaturation dependence of the stationary nucleation rate (schematic representation)

gives the non-stationary nucleation rate $J(n, t), Z_{e}(n, t)$ being the number of $n$-atomic clusters expressed as a quasiequilibrium distribution function:

$Z_{n, e}=\frac{\omega_{+0} \omega_{+1} \omega_{+2} \ldots \omega_{+n-1}}{\omega_{-1} \omega_{-2} \omega_{-3} \ldots \omega_{-n}}=N_{0} \exp \left[-\frac{\Delta \tilde{G}(n)}{k T}\right]$

The differential Eq. (25) has no exact analytical solution but various approximate solutions are known nowadays [18-30]. It was Zeldovich's personal opinion, however, that the continuity approach should not be applied to cases in which critical nuclei consist of only several atoms. ${ }^{3}$

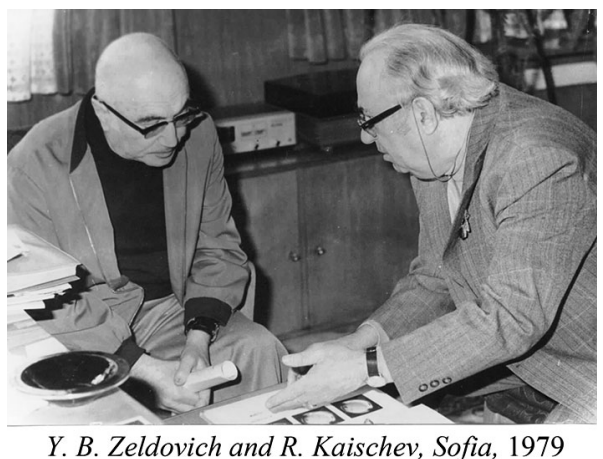

Namely this Zeldovich comment made me look for another explanation of the non-stationary induction times, experimentally observed in case of electrochemical nucleation on a foreign substrate and the next section presents the obtained theoretical and experimental results.

\section{Non-stationary nucleation kinetics on a changing number of active sites}

Electrochemical nucleation on active sites was theoretically and experimentally examined by various authors and

\footnotetext{
${ }^{3}$ I met J.B.Zeldovich and had the chance to talk with him during his visit to the Institute of Physical Chemistry, BAS in April 1979.
} 
a detailed reference list can be found in Ref. [1]. Here I will mention only the pioneering work of Erdey-Gruz and Volmer [31], who were the first to notice that nuclei of the new phase were formed on some preferred sites on the electrode surface, the work of Fleischmann and Thirsk [32], who considered the case of equally active sites and the works of Kaischev and Mutaftschiev [33], Markov et al. [34-36] and Fletcher et al. [37-42], who examined the nucleus formation on active sites with different activities with respect to the process of nucleus formation. Attention should be paid also to the works of Sharifker and Mostany [43], Sluyters-Rehbach et al. [44, 45], Mirkin and Nilov [46], Heerman and Tarallo [47-50] and Danilov et al. [51-53], who contributed essentially to this very important subject, too (see also Ref. [30] and the references cited therein).

It is necessary to note, however, that in most cases the theoretical studies as well as the performed interpretation of experimental results were based on the essential assumption for an initial constant number $N_{0}$ of active sites on the electrode surface. Thus, during the process of nucleus formation the number of free active sites could only decrease for two reasons: the nucleus formation thereon and the spreading of zones of reduced concentration and overpotential around the growing stable clusters since free active sites within such zones were practically deactivated, too. In what follows I will pay attention to the more complex case when free active sites may also appear on the electrode surface due to independent electrochemical reactions, parallel to the process of nucleus formation [54-58] (see also Refs. [1, 30] and the references cited therein).

Consider an electrochemical system comprising a solution of metal ions and an inert working electrode, which at time $t=0$ contains a total number of $N_{0}=Z_{a}^{0}+Z_{0}$ active sites. $Z_{a}^{0} / \mathrm{cm}^{-2}$ of them are available active sites and $Z_{0} /$ $\mathrm{cm}^{-2}$ are latent active sites, which can be developed only after polarizing the working electrode to a potential $E$ more negative than the equilibrium potential $E_{\infty}$. Thus, at time $t$ when a cathodic electrochemical overpotential $\eta=E_{\infty}-E$ is applied to the electrochemical system $N(t)$ nuclei of the new phase are formed on the working electrode and the overall nucleation kinetics is described by the following two differential equations:

$$
\begin{aligned}
& \frac{d Z_{a}}{d t}=K_{a}^{+}\left(N_{0}-Z_{a}-N\right)-\left(K_{a}^{-}+K_{n}\right) Z_{a} \\
& \frac{d N}{d t}=K_{n} Z_{a}
\end{aligned}
$$

Equation (28) describes the time dependence of the number $Z_{a}(t) / \mathrm{cm}^{-2}$ of free active sites on the electrode surface whereas Eq. (29) describes the time dependence of the number $N(t)$ of nuclei formed thereon. $K_{a}^{+} / s^{-1}$ and $K_{a}^{-} /$ $s^{-1}$ are the frequencies of appearance and disappearance of free active sites, respectively, and $K_{n} / s^{-1}$ is the frequency of appearance of nuclei of the new phase on the available free active sites.

Having determined $Z_{a}=\left(1 / K_{n}\right)(\mathrm{d} N / \mathrm{d} t)$ and $\mathrm{d} Z_{a} / \mathrm{d} t=(1 /$ $\left.K_{n}\right)\left(\mathrm{d}^{2} N / \mathrm{d} t^{2}\right)$ from Eq. (29) and substituting the two expressions in Eq. (28) the latter transforms into a secondorder differential equation:

$\frac{d^{2} N}{d t^{2}}+A \frac{d N}{d t}+B\left(N-N_{0}\right)=0$

the solution of which yields the following expression for the number $N(t)$ of nuclei of the new phase formed on the working electrode:

$$
\begin{aligned}
N(t)= & N_{0}\left[1-\frac{A+\lambda-P}{2 \lambda} \exp \left(-\frac{A-\lambda}{2} t\right)+\frac{A-\lambda-P}{2 \lambda}\right. \\
& \left.\times \exp \left(-\frac{A+\lambda}{2} t\right)\right]
\end{aligned}
$$

Correspondingly for the rate of nucleus formation $J(t)=\mathrm{d} N(t) / \mathrm{d} t$ one obtains:

$$
\begin{aligned}
J(t)= & \frac{N_{0} B}{\lambda}\left[\left(1-\frac{P}{A+\lambda}\right) \exp \left(-\frac{A-\lambda}{2} t\right)-\left(1-\frac{P}{A-\lambda}\right)\right. \\
& \left.\times \exp \left(-\frac{A+\lambda}{2} t\right)\right]
\end{aligned}
$$

The constants in Eqs. (30, 31 and 32) read $A=K_{a}^{+}+$ $K_{a}^{-}+K_{n}, B=K_{a}^{+} K_{n}, P=2 K_{n} Z_{a}^{0} / N_{0}$ and $\lambda=\left(A^{2}-4 B\right)^{1 / 2}$ $>0[1,54-58]$.

As seen from Fig. 7 the rate of nucleus formation (line 1) has a non-zero initial value $J(0)=K_{n} Z_{a}^{0}$, a maximal value $J_{m}$ at time $t_{m}$ given by

$t_{m}=\frac{1}{\lambda} \ln \frac{(A-\lambda-P)(A+\lambda)^{2}}{(A+\lambda+P)(A-\lambda)^{2}}$

and tends to zero at sufficiently long times. As for the $N(t)$ relationship (line 2) it starts from zero at $t=0$ and attains the constant value $N_{0}=Z_{a}^{0}+Z_{0}$ at sufficiently long times.

The general theoretical Eq. (31) predicts $N(t)$ relationships similar to the experimental ones shown in Fig. 3 if the inequalities $(A-\lambda) t / 2 \leq 0.01$ and $(A+\lambda) t / 2 \geq 5$ are simultaneously fulfilled within a sufficiently wide time interval $\Delta t_{s}[1,58]$. In that case $\exp [-(A-\lambda) t / 2] \approx$ $1-(A-\lambda) t / 2, \exp [-(A+\lambda) t / 2] \approx 0$ and Eq. (31) turns into the linear relationship $N(t)=I_{\mathrm{st}}\left(t-t_{0}\right)$, its slope $\mathrm{d} N(t) /$ $\mathrm{d} t=I_{\mathrm{st}}$ and intercept $t_{0}$ from the time axis giving the stationary nucleation rate and the induction time as,

$I_{\mathrm{st}}=\frac{N_{0} B(A+\lambda-P)}{\lambda(A+\lambda)}$ 
$t_{0}=\frac{4 B-P(A+\lambda)}{2 B(A+\lambda-P)}$

In the more simple case when the initial number $Z_{a}^{0}$ of free active sites tends to zero, the constant $P$ equals zero, too, and Eqs. (33 and 34) simplify to

$I_{\mathrm{st}}=\frac{Z_{0} B}{\lambda}$

$t_{0}=\frac{2}{(A+\lambda)}$

The performed theoretical considerations clearly show that in the general case the stationary nucleation rate $I_{\mathrm{st}}$ and the induction time $t_{0}$ depend essentially on the three rate constants $K_{a}^{+}, K_{a}^{-}$and $K_{n}$. In what follows I consider briefly three different particular cases in which simpler theoretical expressions are obtained for $I_{\mathrm{st}}$ and $t_{0}$ depending on the values of $K_{a}^{+}, K_{a}^{-}$and $K_{n}$.

$K_{a}^{+} \gg K_{a}^{-}+K_{n}$

In this case, free active sites appear on the electrode surface with a high frequency $K_{a}^{+}$, the slow, rate-determining step of the complex process of new phase formation is the appearance of nuclei on the available free active sites with a frequency $K_{n}$ and Eqs. (33 and 34) transform into:

$I_{\text {st }}=K_{n} N_{0}$

$t_{0}=\frac{1}{K_{a}^{+}}\left(1-\frac{Z_{a}^{0}}{N_{0}}\right)$

Therefore, the slopes $\mathrm{d} N / \mathrm{d} t=I_{\mathrm{st}}$ of the linear parts of the $N$ vs $t$ relationships (Fig. 3) are determined by the nucleation frequency $K_{n}$ at the corresponding overpotential

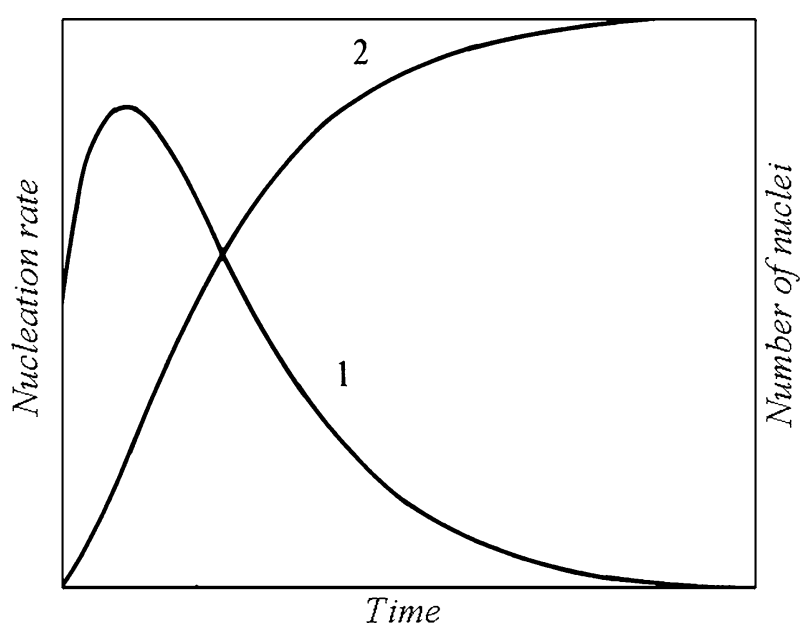

Fig. $7 J(t)$ (line 1) and $N(t)$ (line 2) relationships corresponding to Eqs. (32 and 31) resp., a schematic representation whereas the intercepts $t_{0}$ from the time axis contain information on the frequency $K_{a}^{+}$of appearance of active sites on the electrode surface.

$K_{n} \gg K_{a}^{+}+K_{a}^{-}$

In this case, the slow step of the overall nucleation process is the appearance of free active sites on the electrode surface and, therefore, the slopes $\mathrm{d} N / \mathrm{d} t$ of the linear parts of the $N$ vs $t$ relationships (Fig. 3) are determined by the frequency $K_{a}^{+}$whereas the intercept $t_{0}$ from the time axis depends on the nucleation frequency $K_{n}$ :

$I_{\mathrm{st}}=K_{a}^{+} Z_{0}$

$t_{0}=\frac{1}{K_{n}}\left(1-\frac{K_{n} Z_{a}^{0}}{K_{a}^{+} N_{0}}\right)\left(1-\frac{Z_{a}^{0}}{N_{0}}\right)^{-1}$

$K_{a}^{-} \gg K_{a}^{+}+K_{n}$

Finally, if the frequency $K_{a}^{-}$exceeds essentially the sum of $K_{a}^{+}$and $K_{n}$ for $I_{\text {st }}$ and $t_{0}$ it results:

$I_{\mathrm{st}}=\frac{K_{a}^{+} K_{n}}{K_{a}^{-}} N_{0}$

$t_{0}=\frac{1}{K_{a}^{-}}\left(1-\frac{K_{a}^{-} Z_{a}^{0}}{K_{a}^{+} N_{0}}\right)$

which means that both the slopes $\mathrm{d} N / \mathrm{d} t$ of the linear parts of the $N$ vs $t$ relationships and the intercept $t_{0}$ from the time axis depend essentially on the three rate constants $K_{a}^{+}, K_{a}^{-}$ and $K_{n}$.

\section{Conclusions}

The considerations performed thus far clearly show that nucleation in electrochemical systems is not really a simple process and requires detailed theoretical and experimental studies. This is particularly true when active sites for nucleus formation appear on and disappear from the electrode surface simultaneously with the nuclei of the new phase. In this case it is practically impossible to comprehend and interpret the results of a nucleation experiment if the latter is performed by means of the standard triple pulse potentiostatic technique schematically presented in Fig. 1. To overcome this difficulty we proposed a modified pulse potentiostatic technique [56, 57] allowing us to fix the energy state of the electrode surface and to distinguish the actual rate of nucleus formation from the rate of appearance of active sites thereon. Those who are interested in this subject can find detailed information also in Refs. [1, 58]. 


\section{References}

1. Milchev A (2002) Electrocrystallization: fundamentals of nucleation and growth. Kluwer Academic Publishers, Boston

2. Becker R, Döring W (1935) Ann Phys 24:719

3. Kaischev R (1945/1946) Ann Univ Sofia Fac Physicomat Livre 2 (Chem) 42:109

4. Milchev A, Stoyanov S, Kaischev R (1974) Thin Solid Films $22: 255$

5. Milchev A, Stoyanov S, Kaischev R (1974) Thin Solid Films 22:267

6. Milchev A, Stoyanov S (1976) J Electroanal Chem 72:33

7. Milchev A, Stoyanov S, Kaischev R (1977) Electrokhimia 13:855

8. Milchev A (1991) Contemp Phys A 32:321

9. Milchev A (2015) Nucleation phenomena in electrochemical systems: thermodynamic concepts. ChemTexts. doi:10.1007/ s40828-015-0022-0 (in this issue)

10. Volmer M (1939) Kinetik der Phasenbildung. Theodor Steinkopff Verlag, Leipzig

11. Zeldovich Ya B (1942) Zh Eksp Theor Phys, 12:525 (in Russian); English translation: (1943) Acta Physicohim USSR 18:1

12. Frenkel Ya I (1955) Kinetic theory of liquids. Dover, New York

13. Michailova E, Milchev A (1988) J Appl Electrochem 18:614

14. Milchev A, Zapryanova T (2006) Electrochem Commun 51:2926

15. Walton D (1962) J Chem Phys 37:2182

16. Walton D (1969) Nucleation Zettlemoyer AC (ed) Marcel Dekker Inc, New York, p. 379

17. Stoyanov S (1973) Thin Solid Films 18:91

18. Probstein RF (1951) J Chem Phys 19:619

19. Kantrowitz A (1951) J Chem Phys 19:1097

20. Farley FJ (1952) Proc R Soc 212:530

21. Wakeshima H (1954) J Chem Phys 22:1614

22. Collins FC (1955) Z Electrochem 59:404

23. Chakraverty BK (1966) Surf Sci 4:205

24. Kashchiev D (1969) Surf Sci 14:209

25. Baraboshkin AN (1976) Electrocrystallization of metals from molten salts (in Russian). Nauka, Moscow

26. Roldigin VI, Danilov AI, Polukarov YuM (1985) Russ J Electrochem 21:608
27. Danilov AI, Polukarov YuM (1987) Russ Chem Rev 56:619

28. Isaev VA, Volegov AV, Baraboshkin AN (1989) Rasplavi 3:114

29. Isaev VA (1998) J Electroanal Chem 453:25

30. Isaev VA (2007) Electrochemical phase formation (in Russian). UrO, Russian Academy of Sciences, Ekaterinburg

31. Erdey-Grúz T, Volmer M (1931) Z Phys Chem 157(A):165

32. Fleischmann M, Thirsk HR (1959) Electrochim Acta 1:146

33. Kaischev R, Mutaftschiev B (1965) Electrochim Acta 10:643

34. Markov I, Stoycheva E (1976) Thin Solid Films 35:21

35. Markov I, Kashchiev D (1972) J Cryst Growth 16:170

36. Markov I (1976) Thin Solid Films 35:11

37. Fletcher S, Lwin T (1983) Electrochim Acta 28:237

38. Deutscher RL, Fletcher S (1984) J Electroanal Chem 164:1

39. Fletcher S (1984) J Electroanal Chem 164:11

40. Deutscher RL, Fletcher S (1988) J Electroanal Chem 239:17

41. Deutscher RL, Fletcher S (1990) J Electroanal Chem 277:1

42. Deutscher RL, Fletcher S (1998) J Chem Soc Faraday Trans 94:3527

43. Scharifker BR, Mostany J (1984) J Electroanal Chem 177:13

44. Sluyters-Rehbach M, Wijenberg JHOJ, Bosko E, Slyuters JH (1987) J Electroanal Chem 236:1

45. Wijenberg JHOJ, Mulder WH, Sluyters-Rehbach M, Slyuters JH (1988) J Electroanal Chem 256:1

46. Mirkin MV, Nilov AP (1990) J Electroanal Chem 283:35

47. Heerman L, Tarallo A (1998) J Electroanal Chem 451:101

48. Heerman L, Tarallo A (1998) J Electroanal Chem 455:265

49. Heerman L, Tarallo A (1999) J Electroanal Chem 470:70

50. Heerman L, Tarallo A (2000) Electrochem Commun 2:85

51. Danilov AI, Molodkina EB, Polukarov YuM (2000) Russ J Electrochem 36:916

52. Danilov AI, Molodkina EB, Polukarov YuM (2000) Russ J Electrochem 36:1092

53. Danilov AI, Molodkina EB, Polukarov YuM, Climent V, Feliu JM (2001) Electrochim Acta 46:3173

54. Milchev A (1985) Electrochim Acta 30:125

55. Milchev A (1986) Electrochim Acta 31:977

56. Milchev A (1998) J Electroanal Chem 457:35

57. Milchev A (1998) J Electroanal Chem 457:47

58. Milchev A (2008) Russ J Electrochem 44:619 